\title{
Results from a near infrared search for emission-line stars in the Inner Galaxy: Spectra of new Wolf-Rayet stars ${ }^{\star}$
}

\author{
N. L. Homeier ${ }^{1,2, \star \star}$, R. D. Blum ${ }^{3, \star \star}$, A. Pasquali ${ }^{4}$, P. S. Conti ${ }^{5}$, and A. Damineli ${ }^{6}$ \\ 1 European Southern Observatory, Karl Schwarzschild Str. 2, Garching bei Muenchen, Germany \\ 2 University of Wisconsin, Madison, Sterling Hall, 475 N. Charter St., Madison, WI, USA \\ 3 Cerro Tololo Interamerican Observatory, Casilla 603, La Serena, Chile \\ ${ }^{4}$ European Southern Observatory - Space Telescope European Coordinating Facility, Garching, Germany \\ 5 JILA and APS Department, University of Colorado, Boulder, CO 8030, USA \\ ${ }^{6}$ Universidade de São Paulo, IAG, CP 3386, São Paulo 01060-970, Brazil
}

Received 9 May 2003 / Accepted 25 June 2003

\begin{abstract}
We present follow-up spectroscopy of emission line candidates detected on near-infrared narrow band images in the inner Galaxy (Homeier et al. 2003). The filters are optimized for the detection of Wolf-Rayet stars and other objects which exhibit emission-lines in the $2 \mu \mathrm{m}$ region. Approximately three square degrees along the Galactic plane have been analyzed in seven narrow-filters (four emission-lines and three continuum). We have discovered 4 new Wolf-Rayet stars and present coordinates, finding charts, and $K$-band spectra.
\end{abstract}

Key words. stars: Wolf-Rayet - stars: emission-line - Galaxy: center - Galaxy: stellar content - infrared: stars

\section{Introduction}

Dust in the plane of our Galaxy severely compromises any attempt at complete samples of young stars, but can be mediated by observing at longer wavelengths. In Homeier et al. (2003), we discussed the difficulty in conducting searches for evolved massive stars in the optical passbands, and detailed our observing strategy for finding obscured emission-line stars using narrow band filters near $2 \mu \mathrm{m}$. The eventual goal of such a project is the identification of this young stellar population to increase the number of known Wolf-Rayet (WR) stars, to improve the statistics of WR stars in clusters vs. isolated (likely runaways) WRs, and to use WR stars as a tracer of Galactic structure. In this paper we report on spectroscopic follow-up from our imaging program in the inner Milky Way.

\section{Observations and reduction}

For details of our reduction strategy for the survey images and candidate selection we refer the reader to Homeier et al. (2003). Our candidates are selected with a combination of photometric

Send offprint requests to: N. Homeier,

e-mail: nhomeier@eso.org

* Based on observations made with ESO Telescopes at the La Silla Observatory under programme ID P69.D-0567.

$\star \star$ Visiting Astronomer, Cerro Tololo Inter-American Observatory, National Optical Astronomy Observatories, which are operated by the Association of Universities for Research in Astronomy, Inc., under cooperative agreement with the National Science Foundation. index and image subtraction. We avoided the region within $5^{\prime}$ of the Galactic center due to severe crowding.

In Table 1 we present central wavelengths and FWHMs for our chosen set of $K$-band filters (Blum \& Damineli 1999; Homeier et al. 2003). Four filters are centered on the characteristic WR wind emission lines of He I $2.06 \mu \mathrm{m}$, C IV $2.08 \mu \mathrm{m}$, $\mathrm{H} \mathrm{I} \mathrm{Br} \gamma 2.166 \mu \mathrm{m}$, and He II $2.189 \mu \mathrm{m}$, and the additional three continuum filters are at $2.03 \mu \mathrm{m}, 2.14 \mu \mathrm{m}$, and $2.248 \mu \mathrm{m}$. Thus each line filter measurement has a continuum point to the red and blue, which accounts for spatially variable extinction.

Spectra were taken with SOFI (Moorwood et al. 1998) on the European Southern Observatory's (ESO) $3.6 \mathrm{~m} \mathrm{New}$ Technology Telescope (NTT) at La Silla on the nights of 2002 May 1-2, 4-6 and with OSIRIS ${ }^{1}$ on the Cerro Tololo InterAmerican Observatory's (CTIO) Blanco $4 \mathrm{~m}$ during the nights of 2000 July $12-13$ and 2002 July $9-12$. Seeing varied between $0.8^{\prime \prime}$ and $2^{\prime \prime}$ during the run at the NTT, between 1-1.5" during the 2000 run at the Blanco $4 \mathrm{~m}$, and between $0.8^{\prime \prime}$ and $1.5^{\prime \prime}$ during the 2002 run at the Blanco $4 \mathrm{~m}$.

SOFI and OSIRIS deliver plate scales of $0.29^{\prime \prime} \mathrm{pixel}^{-1}$ and $0.40^{\prime \prime}$ pixel $^{-1}$, respectively. With SOFI we used the GR Grism Red for a wavelength coverage from $1.5-2.4 \mu \mathrm{m}$, and the $f / 3$

\footnotetext{
${ }^{1}$ OSIRIS (Ohio State Infrared Imager and Spectrograph) is a collaborative project between Ohio State University and CTIO. Osiris was developed through NSF grants AST 90-16112 and AST 9218449. OSIRIS is described in the instrument manuals found on the CTIO Web site at http://www.ctio.noao.edu. See also DePoy et al. (1993).
} 
Table 1. Filter description.

\begin{tabular}{cc}
\hline \hline Central $\lambda(\mu \mathrm{m})$ & $F W H M(\mu \mathrm{m})$ \\
\hline 2.032 & 0.010 \\
2.062 & 0.010 \\
2.077 & 0.015 \\
2.142 & 0.020 \\
2.161 & 0.022 \\
2.191 & 0.013 \\
2.248 & 0.024 \\
\hline
\end{tabular}

Table 2.

\begin{tabular}{ccc}
\hline \hline Star & RA (J2000) & Dec (J2000) \\
\hline WR 101p & $17: 45: 42.47$ & $-28: 52: 53.3$ \\
WR 101q & $17: 45: 57.78$ & $-28: 54: 46.1$ \\
WR 102ca & $17: 46: 13.04$ & $-28: 49: 25.4$ \\
WR 102ka & $17: 46: 18.12$ & $-29: 01: 36.5$ \\
\hline
\end{tabular}

spectroscopic mode on OSIRIS for a wavelength coverage of $2.0-2.4 \mu \mathrm{m}$. The spectral resolution at $2.2 \mathrm{~m}$ is $\lambda / \delta \lambda \approx 800$ for SOFI and $\approx 1200$ for OSIRIS.

We offset our targets by $5^{\prime \prime}$ along the slit and subtracted these paired observations from each other to remove the background. Spectra were extracted with the APALL task in IRAF ${ }^{2}$. $\mathrm{A} 0-2 \mathrm{~V}$ stars were observed at a range of airmass, the $\mathrm{Br} \gamma$ line was removed by linear interpolation, and telluric correction of the science spectra was performed with these spectra. For the NTT observations, wavelength calibration was performed using arc spectra. For the 2000 Blanco observations, wavelength calibration was accomplished using $\mathrm{OH}$ emission lines (Oliva \& Origlia 1992). For the 2002 Blanco observations, rough wavelength calibration was accomplished using telluric features in the standard star spectrum. Finally, the science spectra were normalized to the continuum.

\section{Results: 4 new Wolf-Rayet stars}

We have discovered four Wolf-Rayet stars: three WC and one WN. Names were given to the stars following the convention in the Seventh Catalog of Wolf-Rayet Stars (van der Hucht 2001). In Table 3 we list coordinates for the four stars and in Figs. 1-8 we present spectra and finding charts.

\subsection{Spectra}

The three WC stars, WR 101p, WR 101q, and WR 102ca, have nearly identical 2-2.4 $\mu \mathrm{m}$ spectra. The most significant differences are: (1) WR 101p lacks the C IV line at $2.139 \mu \mathrm{m}$, (2) WR 101q has a C III line at $\sim 2.11 \mu \mathrm{m}$ which is stronger than the blend of C IV lines at $2.08 \mu \mathrm{m}$, in contrast to WR 101p and WR 102ca, (3) WR 101q has a line at $2.22 \mu \mathrm{m}$ of an unknown transition, and (4) WR 102ca appears to have more dust than WR 101p and WR 101q, as its lines are weaker overall due to excess continuum emission by dust; see the discussion below on excess emission.

\footnotetext{
${ }^{2}$ IRAF is distributed by the National Optical Astronomy Observatories.
}

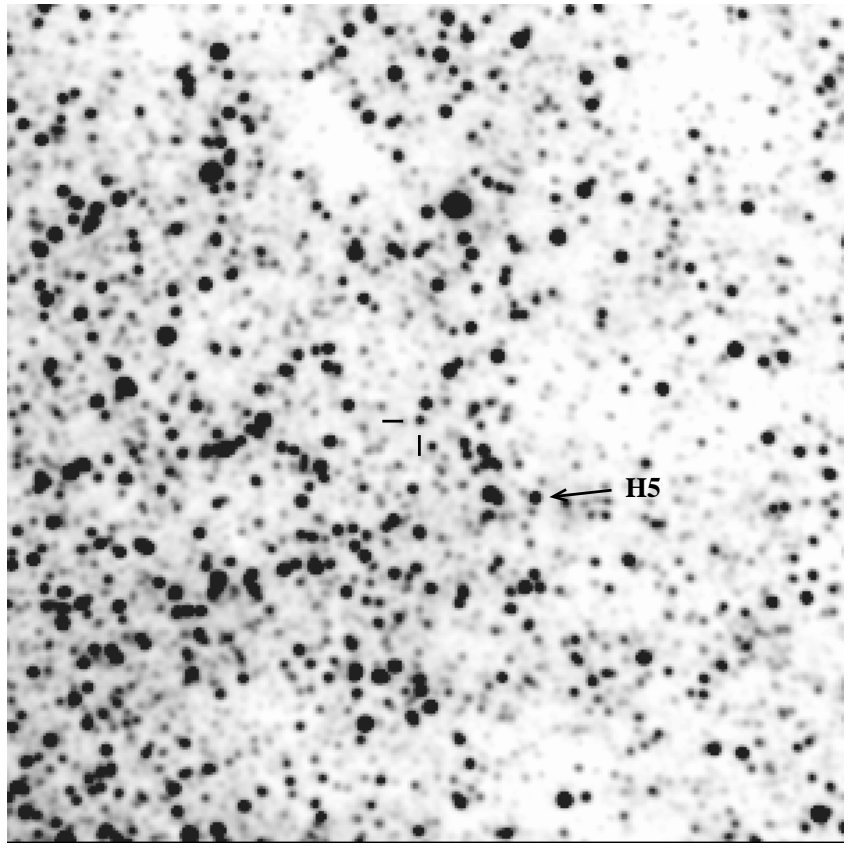

Fig. 1. Finding chart for WR 101p. The star H5 from Cotera et al. (1999) is $\sim 51^{\prime \prime}$ to the Southwest. North is up and east is to the left. The image size is $5 \times 5^{\prime}$.

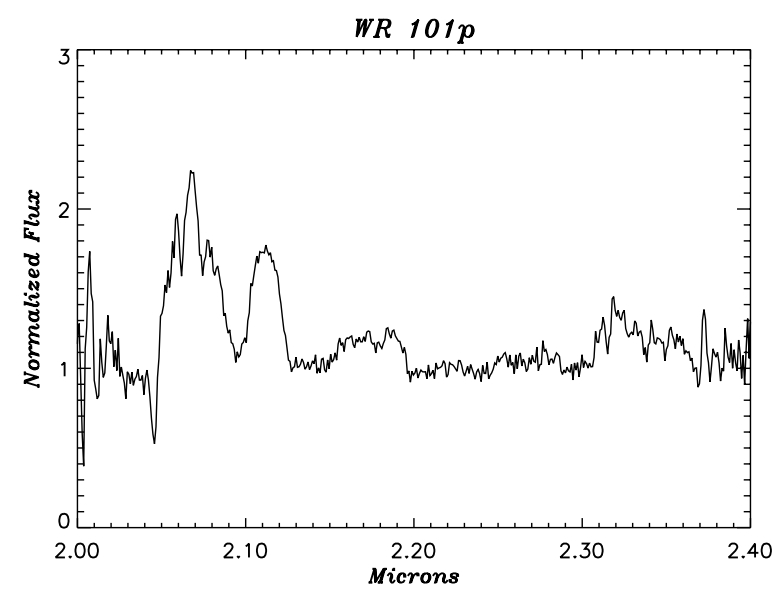

Fig. 2. Spectrum of WR 101p.

The spectra are similar to the spectrum of star 309 in the Quintuplet cluster, which Figer et al. (1999) classified as earlier than WC8 due to its excess emission from He II $3.09 \mu \mathrm{m}$ and lack of strong emission from He I $2.058 \mu \mathrm{m}$. Based on their similar spectral morphology in the $2-2.4 \mu \mathrm{m}$ region, these spectra are consistent with the same classification, with varying amounts of dust dilution. Of the published spectra of known WRs other than the Quintuplet WRs, the closest morphological match is the WC9 star WR 88 (Eenens et al. 1991). We therefore classify these stars as WC8-9.

The 2-2.4 $\mu \mathrm{m}$ spectrum of WR 102ka shows strong lines of $\mathrm{He}$ I $2.06 \mu \mathrm{m}$ and $\mathrm{Br} \gamma$, weak lines at $2.103 \mu \mathrm{m} \mathrm{C} \mathrm{III/N} \mathrm{III,}$ $2.114 \mu \mathrm{m} \mathrm{He} \mathrm{I/N} \mathrm{III,} 2.189 \mu \mathrm{m}$ He II, and possibly an unidentified feature at $2.139 \mu \mathrm{m}$. In Fig. 9 we present the $2.05-2.25 \mu \mathrm{m}$ region of the spectrum of WR $102 \mathrm{ka}$ to illustrate these weak features. 
Table 3. 2MASS magnitudes.

\begin{tabular}{ccccccccc}
\hline \hline Name & $J$ & error & $H$ & error & $K_{\mathrm{s}}$ & error & $J-H$ & $H-K_{\mathrm{s}}$ \\
\hline WR 101p & 16.32 & 0.132 & 13.05 & 0.059 & 11.20 & 0.049 & 3.27 & 1.85 \\
WR 101q & 16.26 & null & 13.47 & 0.070 & 11.49 & 0.056 & 2.79 & 1.98 \\
WR 102ca & 14.56 & null & 12.79 & 0.071 & 10.40 & 0.075 & 1.77 & 2.39 \\
WR 102ka & 12.98 & 0.022 & 10.27 & 0.025 & 8.84 & 0.031 & 2.71 & 1.43 \\
\hline
\end{tabular}

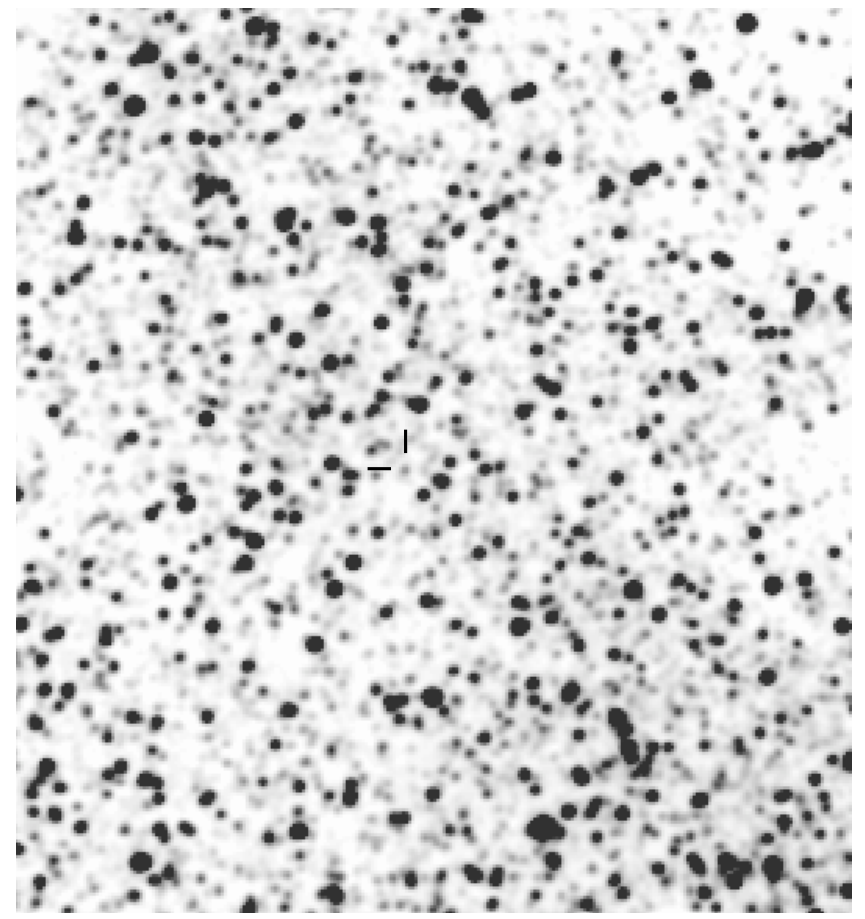

Fig. 3. Finding chart for WR 101q. North is up and east is to the left. The image size is $5 \times 5^{\prime}$.

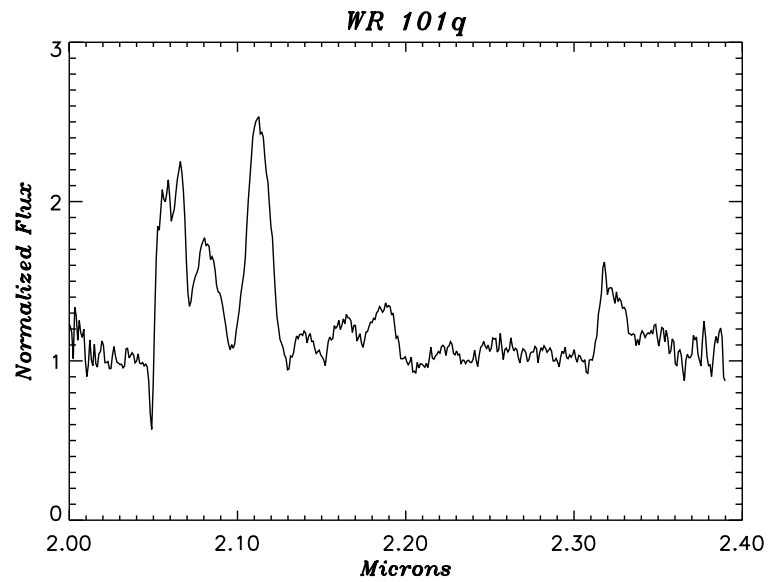

Fig. 4. Spectrum of WR 101q.

Of note is the strength of $\mathrm{He} \mathrm{I} 2.06 \mu \mathrm{m}$ to $\mathrm{Br} \gamma$; the $\mathrm{He} \mathrm{I} / \mathrm{Br} \gamma$ ratio is $\sim 2$. A few of the Galactic Center He I stars have similar He I/Br $\gamma$ ratios (Allen et al. 1990; Krabbe et al. 1991; Blum et al. 1995; Tamblyn et al. 1996; Najarro et al. 1997), but most also have stronger $2.11 \mu \mathrm{m}$ features than WR $102 \mathrm{ka}$. The most similar spectral morphology is displayed by two LMC stars, BAT99 45 (HDE 269582, BE 294) and

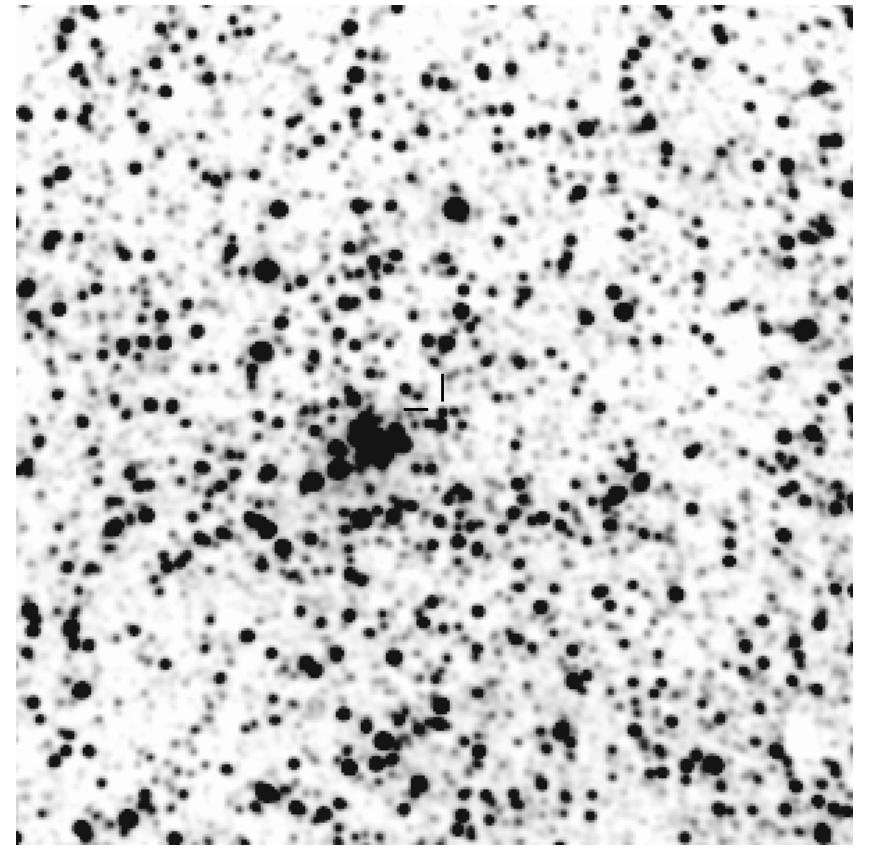

Fig. 5. Finding chart for WR 102ca. The concentration of stars is the Quintuplet cluster, and WR 102ca is a member. North is up and east is to the left. The image size is $5 \times 5^{\prime}$.

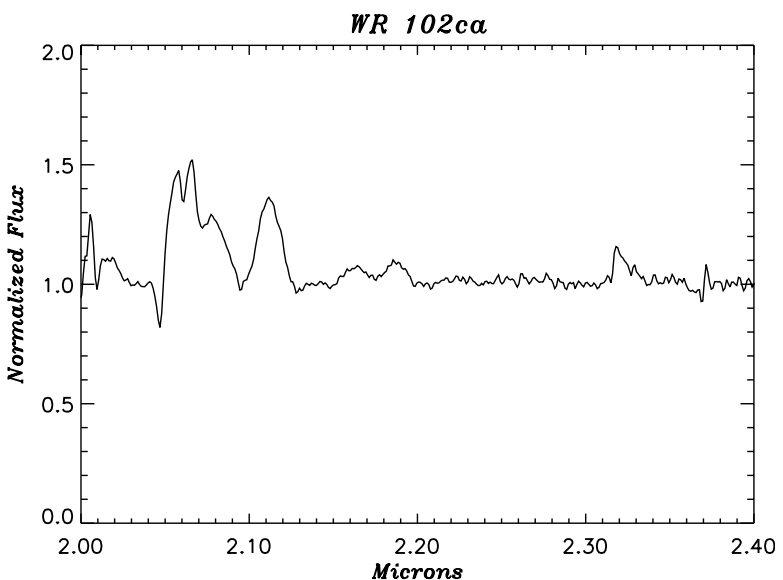

Fig. 6. Spectrum of WR 102ca.

BAT99 13 (Hen S9, Sk -66 40) (McGregor et al. 1988; Breysacher et al. 1999). These stars were originally classified as Ofpe/WN9, but later re-classified as WN10h and WN10 (see Crowther \& Smith 1997 for an explanation). The general picture is that stars between $40-60 M_{\odot}$ evolve through an LBV stage to become WN8 stars, and in their dormant stage are identified as WN9 - 11 stars (Crowther \& Smith 1997; Pasquali et al. 1999). The star BAT99 45 is at least 


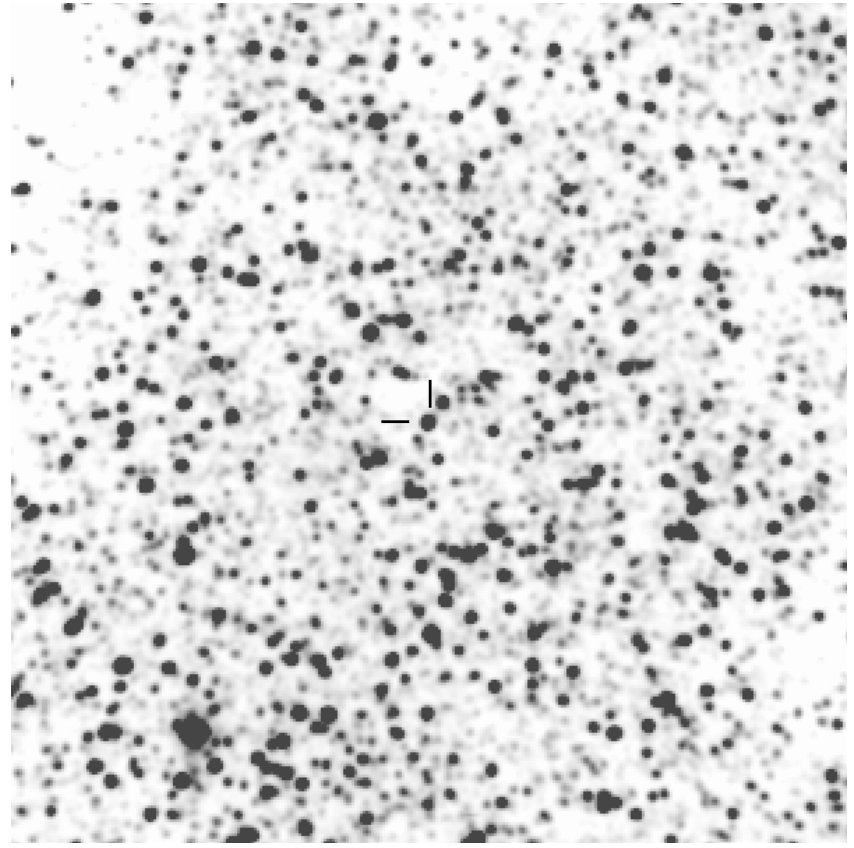

Fig. 7. Finding chart for WR $102 \mathrm{ka}$. North is up and east is to the left. The image size is $5 \times 5^{\prime}$.

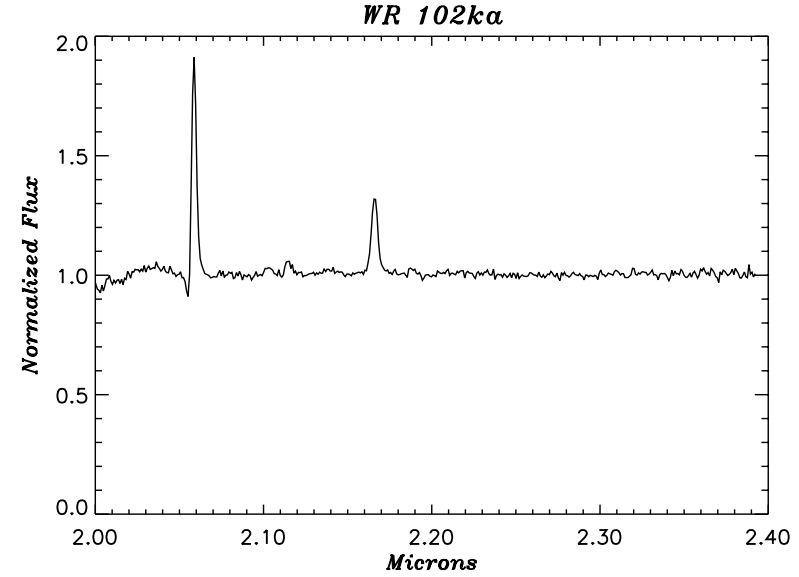

Fig. 8. Spectrum of WR 102ka.

spectrally variable. The $\mathrm{HeI} / \mathrm{Br} \gamma$ strength changed by a factor of 10 between 1987 January and 1993 September (McGregor et al. 1988; Blum et al. 1995). Due to the similarity of WR 102ka's 2-2.4 $\mu \mathrm{m}$ spectra with BAT99 45 and BAT99 13, we classify it as WN10.

\section{2. $J H K_{s}$ photometry}

In Table 3 we present $J H K_{\mathrm{s}}$ magnitudes from the latest calibration of the Two Micron All-Sky Survey (2MASS) cata$\log ^{3}$ A $J-H$ vs. $H-K_{\text {s }}$ plot is presented in Fig. 10, with the main sequence and giant tracks as solid and dotted lines (Koornneef 1983), and the reddening line for the bluest stars as a dashed line according to a Rieke \& Lebofsky (1985) extinction law. Objects falling to the right of the reddening line have colors inconsistent with extinction along the line of sight

\footnotetext{
${ }^{3}$ http://www.ipac.caltech.edu/2mass/
}

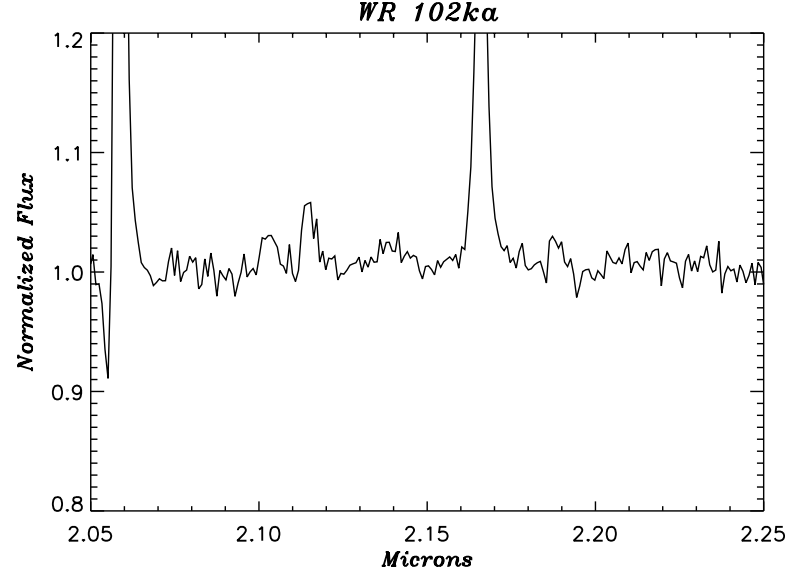

Fig. 9. Spectrum of WR 102ka on a different scale.

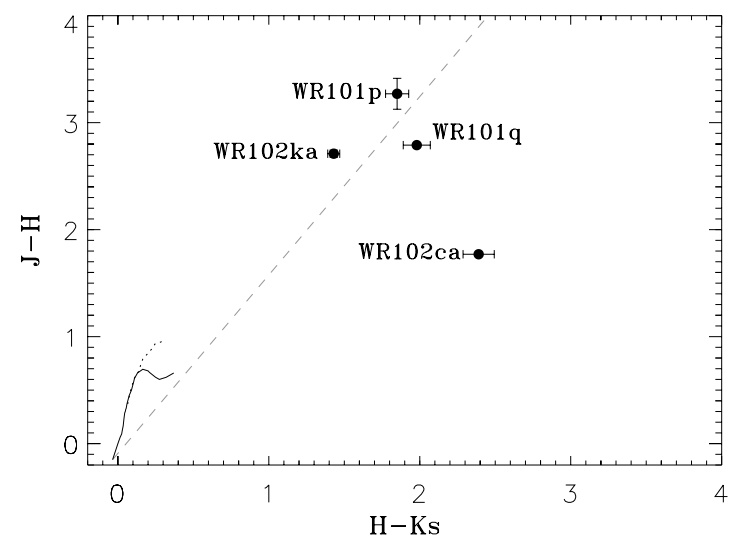

Fig. 10. $J-H$ vs. $H-K_{\text {s }}$ plot with magnitudes taken from the 2MASS catalog; see text. The main sequence and giant tracks are indicated by the solid line and dotted lines (Koornneef 1983) and the reddening line is overplotted as the dashed line (1985).

for the bluest stars, i.e. they have excess emission at $K_{\mathrm{s}}$, usually attributable to hot dust.

$J, H$, and $K_{\mathrm{s}}$ errors were added in quadrature to produce the error bars in Fig. 10. For WR 101q and WR 102ca, no $J$ errors were listed in the 2MASS catalog, and we have therefore not shown a $J-H$ error.

In Fig. 11 we plot $J-H$ vs. $H-K_{\mathrm{s}}$ values for most of the known Wolf-Rayet stars in the Galaxy taken from the 2MASS catalog. The $J, H, K_{\mathrm{s}}$ magnitudes were retrieved using the positions given in the Seventh Catalog of Wolf-Rayet Stars (van der Hucht 2001). Magnitudes are included if the positions in the 2MASS catalog were within 3 " of the input coordinates. If more than one object was found, the nearest object was taken; misidentifications are possible. We also plot $J-H$ and $H-K^{\prime}$ for the $\mathrm{WN}$ and $\mathrm{WC}$ stars in the Quintuplet cluster, and the four stars with featureless $K$-band spectra referred to as "cocoon stars", or "dusty, late-type WC stars" (DWCLs; Figer et al. 1999). For reference, the $K, K_{\mathrm{s}}$, and $K^{\prime}$ filters have slightly different wavelength coverages: the $K_{\mathrm{s}}$ filter transmission profile starts $\sim 0.04 \mu \mathrm{m}$ bluer and ends $\sim 0.06 \mu \mathrm{m}$ bluer than $K ; K^{\prime}$ starts $\sim 0.04 \mu \mathrm{m}$ bluer and ends $\sim 0.02 \mu \mathrm{m}$ bluer than $K_{\mathrm{s}}$. 


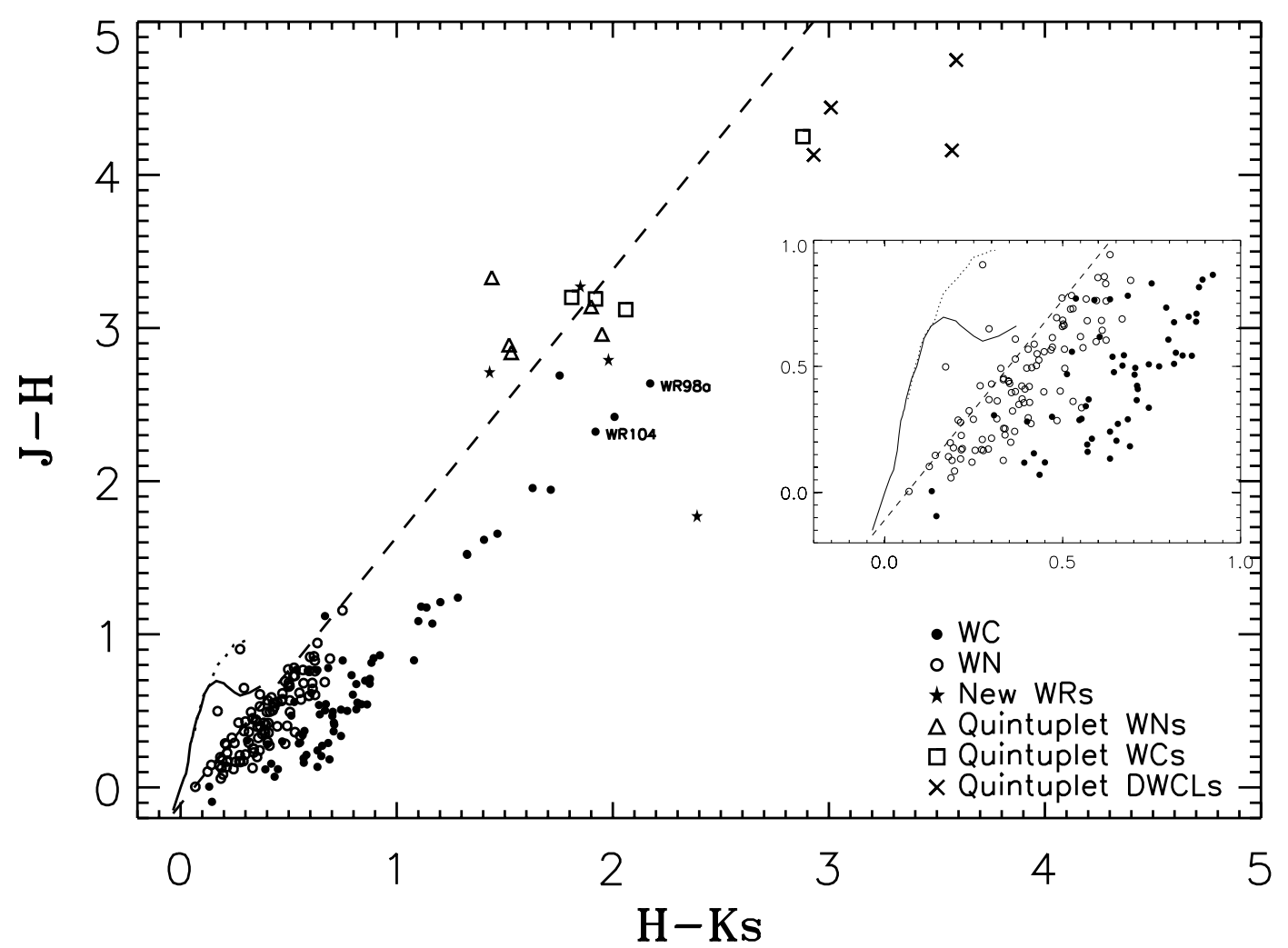

Fig. 11. $J-H$ vs. $H-K_{\text {s }}$ plot for a sample of Galactic WN and WC stars, the stars discovered here, and the Quintuplet WN, WC, and DWCL stars. The $J, H, K_{\mathrm{s}}$ magnitudes were retrieved from the 2MASS catalog using the positions given in the Seventh Catalog of Wolf-Rayet stars (van der Hucht 2001). Magnitudes are included if the positions in the 2MASS catalog were within 3 " of the input coordinates. If more than one object was found, the closest was taken; misidentifications may be possible. $J, H$, and $K^{\prime}$ magnitudes for the Quintuplet stars were taken from Figer et al. (1999); where two measurements were made, only the first is plotted. The main sequence and giant tracks are indicated by the solid line and dotted lines (Koornneef 1983) and the reddening line is overplotted as the dashed line (1985).

The WR stars are offset from the reddening line for the bluest main sequence stars towards larger $H-K_{\mathrm{s}}$. To further illustrate this, we include an inset of this region in Fig. 11. Both the WN and WC subtypes follow sequences parallel to the plotted reddening line due to intervening interstellar dust, with the WN stars offset by $0.1-0.2$ mag and the WC stars by another 0.4 magnitudes. This can be understood as the effect of the contributions from free-free emission in their ionized winds and a blackbody spectrum from hot dust around most WC stars (see Cohen et al. 1975 and references therein), and possibly line emission. WR stars have strong emission lines in their NIR spectra and this may affect their broad-band colors.

We have also labeled the positions of WR 98a and WR 104 in Fig. 11, both which are associated with "pinweel" nebulae (Tuthill et al. 1999; Monnier et al. 1999). The known pinwheel nebulae arise from the interaction of a WC wind with that of a massive companion. The positions of these stars along the WC sequence support the idea that the near-infrared colors are driven by hot dust, and that most WC stars in the Galaxy produce dust (although not all, as some fall in the region occupied by the WN stars).

WR 101p (WC8-9) and WR 102ka (WN10) have nearinfrared colors more typical of Galactic WN stars and most of the Quintuplet WR stars, as does WR 101q (WC8-9), although its $J-H$ color is uncertain due to an unquantified error in its
$J$ magnitude. WR 102ca (WC8-9) appears to have a large infrared excess and at the same time appears to suffer from less extinction than the rest of the Quintuplet WR stars. We note that it is likely that the $J$ magnitude is severely in error, which could give it an anomalously blue $J-H$ color and an overestimated $K$-band excess. In the 2MASS $H$ and $K_{\mathrm{s}}$ images there are two stars within $\sim 0.08^{\prime \prime}$ radius of WR $102 \mathrm{ca}$, while in the $J$ image only one of the three stars (not WR 102ca) is easily visible. All three stars have listings of "null" for their estimated $J$ error, and thus their $J$ magnitudes is likely inaccurate.

\section{Null detections}

In this section we briefly discuss the candidates which turned out not to be emission-line stars upon follow-up spectroscopy. These stars were readily identified as giants by their strong $\mathrm{CO}$ absorption lines. Only the 2.06 and $2.08 \mu \mathrm{m}$ line indices were used successfully to identify WR stars. It is unclear if this is due to a lack of early-type WNs which dominate at the lines of 2.17 and $2.19 \mu \mathrm{m}$, a sensitivity issue due to their typically weaker lines, or a detection bias. Our 2.17 and $2.19 \mu \mathrm{m}$ line indices had considerably more scatter than the 2.06 and $2.08 \mu \mathrm{m}$ counterparts, leading us to conclude that a detection bias is highly likely. We do however note that we were able to use the $2.17 \mu \mathrm{m}$ filter images to identify several objects with nebular Br $\gamma$ emission. 
During the first set of follow-up observations, objects were selected purely on the photometric indices. Due to the large scatter in these indices, the success rate was low (3 emissionline objects out of 45 candidates, including $1 \mathrm{WR}$ star). Other problems included bad pixels and residual images from bright stars. However, this was largely eliminated with the image subtraction step. With the inclusion of this step, we were able to identify 6 emission-line objects, including 3 WR stars, out of 38 candidates. We found that selection as a photometric candidate and a positive identification as a candidate after image subtraction were separately necessary but not sufficient conditions for the identification of an emission-line star.

Of the candidates which were not emission-line objects, most were multiple objects when observed at higher angular resolution while positioning the slit. None of the members of these multiple objects were emission-line stars. All 4 of the identified WRs were apparently isolated at higher angular resolution. This is a clear case for the need for observations at higher angular resolution in the inner Galaxy where crowding is the dominant source of photometric error.

\section{Discussion}

\subsection{WC stars}

All three of the WC stars detected have similar late-type WC spectra (WC8-9) with dilution by dust. WR 102ca is in the Quintuplet cluster, which has a total of 5 members (including this new one) with similar spectra. It also contains 4 stars with featureless $K$-band spectra, the so-called "cocoon" stars, (Figer et al. 1999), which are likely also to be late-type WCs with sufficient dust that the continuum emission overwhelms the lines (Chiar \& Tielens 2001; Moneti et al. 2001).

Dust around WC stars arises in regions of high density, which can be achieved in the wind-wind interaction zone with a massive companion. This has been observationally confirmed; there are known periodic WC dust-producers where the dust is produced at periastron (Williams et al. 1987; Veen et al. 1998b), and recently, the persistent dust-producers WR 104 and WR 98a have been shown to have pinwheelshaped nebula (Tuthill et al. 1999; Monnier et al. 1999) produced by a short-period ( $\sim 1 \mathrm{yr})$ WR-O binary system. It is not known if single WC stars can also produce dust solely through their clumped winds (see Veen et al. 1998a for a discussion). The hint of copius dusty WCs in the inner Galaxy where the metallicity is likely $\geq Z_{\odot}$ may be a clue to required conditions for dust formation around WC stars.

\subsection{WRs in the inner Galaxy}

In Homeier et al. (2003), an estimate for the number of WRs expected in the inner Galactic sample was given, following from a simple smooth model for the distribution of stars and gas in the Milky Way. They concluded that $\sim 28$ WR stars should be detected within the area covered by the imaging observations in the inner Milky Way. The model used for the estimate given in Homeier et al. (2003) was normalized to the local number density of WR stars assumed, and also depends on the scale length of the Galactic disk, and the distribution of WR absolute $K$ magnitudes.

A census of WR stars in the region covered by our images (but outside the Galactic center) shows that there are 35 known WRs in the Arches cluster (Nagata et al. 1995; Cotera et al. 1996; Blum et al. 2001; Figer et al. 2002), 10 in the Quintuplet cluster (Figer et al. 1999), and at least 2 apparently isolated WR stars near Sgr A (Cotera et al. 1999), for a total of 47. Most of these are brighter than $K=12$, which was the limit considered in the estimate. Already we see that the number of known WRs exceeds the prediction by this very simple model by nearly a factor of two. This suggests a higher star formation rate in the inner Galaxy compared to the local neighborhood. Near-infrared observations toward the inner Galaxy have established that this is indeed the case; see for example the review of the Galactic Center by Morris \& Serabyn (1996).

From this calculation, we might expect that no new WRs would be found brighter than $K=12$, whereas we have identified 4 in this region. The addition of these 4 stars is not numerically significant, but 3 of the 4 are notable for their apparently isolated location. Either these stars are isolated and are thus runaways, or they are the most massive object of an association which has not yet been discovered. We find no evidence in our images for associations or clusters around these stars, but given our poor angular resolution, we cannot rule out their existence. Also, small clusters may not live very long near the Galactic center. Numerical simulations predict that small $\left(\leq 2 \times 10^{4} M_{\odot}\right)$ clusters will disperse sufficiently within a few Myr so that their surface densities will be indisinguishable from that of their surroundings (Kim et al. 2000; Portegies Zwart et al. 2002).

\section{Conclusions}

We have discovered 4 new WR stars in the inner Galaxy: three WC8 - 9 stars with similar spectra and one WN10 star. One of the WC stars is a member of the Quintuplet cluster, while the others are not members of any previously known clusters or associations, nor did we find evidence for any previously unknown clusters or associations. We conclude that narrow-band observations at $K$-band wavelengths are a potentially efficient way to identify evolved massive stars in the plane of the Milky Way, and further higher angular resolution work is highly desirable.

Acknowledgements. We would like to thank P. Crowther for especially useful discussions pertaining to WC9 stars and the ambiguity of Ofpe/WN9 $K$-band spectra. N. H. happily acknowledges the ESO Studentship Programme and thanks the University of Wisconsin Graduate School for partial support. We would like to thank the referee for a careful reading and helpful comments. This research has made use of the NASA/IPAC Infrared Science Archive, which is operated by the Jet Propulsion Laboratory, California Institute of Technology, under contract with the National Aeronautics and Space Administration.

\section{References}

Allen, D. A., Hyland, A. R., \& Hillier, D. J. 1990, MNRAS, 244, 706

Blum, R. D., DePoy, D. L., \& Sellgren, K. 1995, ApJ, 441, 603 
Blum, R. D., \& Damineli, A. 1999a, in Wolf-Rayet Phenomena in Massive Stars and Starburst Galaxies, Proc. IAU 193, ed. K. A. van der Hucht, G. Koenigsberger, \& P. R. J. Eenens (San Francisco: ASP), 472

Blum, R. D., Schaerer, D., Pasquali, A., et al. 2001, AJ, 122, 1875

Breysacher, J., Azzopardi, M., \& Testor, G. 1999, A\&AS, 137, 117

Chiar, J. E., \& Tielens, A. G. G. M. 2001, ApJ, 550, L207

Cohen, M., Barlow, M. J., \& Kuhi, L. V. 1975, A\&A, 40, 291

Cotera, A. S., Erickson, E. F., Simpson, J. P., et al. 1996, ApJ, 461, 750

Cotera, A. S., Simpson, J. P., Erickson, E. F., et al. 1999, ApJ, 510, 747

Crowther, P. A., \& Smith, L. J. 1997, A\&A, 320, 500

Depoy, D. L., Atwood, B., Byard, P. L., Frogel, J., \& O’Brien, T. P. 1993, Proc. SPIE, 1946, 667

Eenens, P. R. J., Williams, P. M., \& Wade, R. 1991, MNRAS, 252, 300

Figer, D. F., McLean, I. S., \& Morris, M. 1999, ApJ, 514, 202

Figer, D. F., Najarro, F., Gilmore, D., et al. 2002, ApJ, 581, 258

Homeier, N. L., Blum, R. D., Conti, P. S., \& Damineli, A. 2003, A\&A, 397, 585

Kim, S. S., Figer, D. F., Lee, H. M., \& Morris, M. 2000, ApJ, 545, 301

Krabbe, A., Genzel, R., Drapatz, S., \& Rotaciuc, V. 1991, ApJ, 382, L19

Koornneef, J. 1983, A\&A, 128, 84
McGregor, P. J., Hillier, D., J., \& Hyland, A. R. 1988, ApJ, 334, 639

Moneti, A., Stolovy, S., Blommaert, J. A. D. L., Figer, D. F., \& Najarro, F. 2001, A\&A, 366, 106

Monnier, J. D., Tuthill, P. G., \& Danchi, W. C. 1999, ApJ, 525, L97

Moorwood, A., Cuby, J. G., \& Lidman, C. 1998, The Messenger, 91, 9 Morris, M., \& Serabyn, E. 1996, ARA\&A, 34, 645

Nagata, T., Woodward, C. E., Shure, M., \& Kobayashi, N. 1995, AJ, 109, 1676

Najarro, F., Krabbe, A., Genzel, R., et al. 1997, A\&A, 325, 700

Oliva, E., \& Origlia, L. 1992, A\&A, 254, 466

Pasquali, A., Nota, A., \& Clampin, M. 1999, A\&A, 343, 536

Portegies Zwart, S. F., Makino, J., McMillan, S. L. W., \& Hut, P. 2002, ApJ, 565, 265

Rieke, G. H., \& Lebofsky, M. J. 1985, ApJ, 288, 618

Tamblyn, P., Rieke, G. H., Hanson, M. M., et al. 1996, ApJ, 456, 206

Tuthill, P. G., Monnier, J. D., \& Danchi, W. C. 1999, Nature, 398, 487

van der Hucht, K. A. 2001, VizieR Online Data Catalog, 3215

Veen, P. M., van Genderen, A. M., van der Hucht, K. A., et al. 1998, A\&A, 329, 199

Veen, P. M., van der Hucht, K. A., Williams, P. M., et al. 1998, A\&A, 339, L45

Williams, P. M., van der Hucht, K. A., \& The, P. S. 1987, QJRAS, 28 , 248 